\title{
Use of Maltose Hydrolysis Measurements to Characterize the Interaction between the Aqueous Diffusion Barrier and the Epithelium in the Rat Jejunum
}

\author{
Michael D. Levitt, ${ }^{\star}$ Caryn Fine, ${ }^{\star}$ Julie K. Furne, ${ }^{\star}$ and David G. Levitt ${ }^{\ddagger}$ \\ * Research and Medicine Services, Department of Veterans Affairs Medical Center, Minneapolis, Minnesota 55417; and ${ }^{\ddagger}$ University of \\ Minnesota, Minneapolis, Minnesota 55455
}

\begin{abstract}
Rates of intestinal absorption and surface hydrolysis are determined by the interaction of two barriers: poorly stirred fluid adjacent to the mucosa, and the epithelial cell. These two barriers commonly are modeled as a fixed, flat layer of epithelium covered by a fixed thickness of unstirred fluid. To more accurately simulate these barriers in a villous mucosa, maltase activity (measured in vitro) was distributed over an anatomically correct model of rat jejunal villi. We then determined what interaction of the aqueous and epithelial barriers best predicted in vivo maltose hydrolysis rates measured over a broad range of infusate concentrations. Hydrolysis was accurately predicted by a model in which unstirred fluid extended from $20 \mu \mathrm{m}$ over the villous tips throughout the intervillous space. In this model, the depth of diffusion into the intervillous space is inversely proportional to the efficiency of epithelial handling of the solute. As a result, both the aqueous barrier and the functional surface area are variables rather than constants. Some implications of our findings (relative to the conventional model) include: higher predicted $\mathrm{V}_{\max }$, efficient handling of low concentrations of a solute at the villous tips while high concentrations must penetrate thick aqueous barriers, and sensitive regulation of transport rates via ease of access to the intervillous space. (J. Clin. Invest. 1996. 97: 2308-2315.) Key words: intestinal transport • maltose hydrolysis $\bullet$ unstirred layer $\bullet$ diffusion $\bullet$ motility
\end{abstract}

\section{Introduction}

Intestinal absorption of solute requires that the compound cross two barriers in series: an aqueous diffusion barrier consisting of poorly stirred luminal fluid adjacent to the brush border (the "unstirred layer") and the epithelial cell (1). Knowledge of how these two resistance barriers interact is fundamental to understanding the absorptive process (2). If diffusion across poorly stirred luminal fluid limits solute uptake, absorption can only be altered by changes in luminal stirring. On the other hand, if movement across the epithelial cell is rate limiting, alteration of absorption rate requires modification of epithelial cell surface area and/or the transport efficiency of the individual cell.

Address correspondence to Michael D. Levitt, ACOS for Research, Veterans Administration Medical Center, One Veterans Drive, Minneapolis, MN 55417. Phone: 612-725-2033; FAX: 612-725-2093.

Received for publication 1 November 1995 and accepted in revised form 21 February 1996.

The Journal of Clinical Investigation

Volume 97, Number 10, May, 1996, 2308-2315
The relationship between the epithelium and the aqueous barrier commonly has been modeled as a smooth layer of absorptive cells covered by a uniform thickness of totally unstirred water (2-6). If this simple model were correct, the aqueous diffusion barrier could be represented as a single thickness of water through which all solutes would have to diffuse before absorption. However, the intestinal mucosa has a villous surface. To the extent that transport occurs along the sides, as well as at the tips, of the villi, the simple conventional model seemingly could not accurately reflect the relationship of the two absorption barriers.

The goal of the present study was to use in vivo measurements of maltose hydrolysis to elucidate the true interaction that occurs between the unstirred layer and the epithelium during the surface hydrolysis of maltose in the rat jejunum.

\section{Methods}

Surgical procedure. Male Sprague-Dawley rats (300-350 g; Harlan Sprague-Dawley Inc., Indianapolis, IN) were used in all studies. Under pentobarbital sodium anesthesia, a midline laparotomy was performed and a $15-\mathrm{cm}$ segment of proximal jejunum was identified. A polyethylene cannula $(0.76 \mathrm{~mm}$ i.d.) with a flared tip was inserted into the proximal end of this segment and secured with a purse-string suture. A second cannula (1.67 mm i.d.) was similarly inserted $15 \mathrm{~cm}$ distally. Both cannulas were glued to a $0.5 \times 0.5 \mathrm{~cm}$ sheet of mesh near the insertion sites to prevent the tubes from being drawn into the gut. A trocar was inserted into the posterior abdominal wall and directed subcutaneously to exit at the back of the neck. The cannulas were inserted through the trocar, which was then removed. The jejunal segment was replaced in the abdominal cavity and the abdominal wound was closed. The animals were provided with a glucose-water solution containing acetaminophen for $24 \mathrm{~h}$ and then fed standard rat chow ad lib. The animals were allowed to recover for at least $2 \mathrm{wk}$ before instituting perfusion studies.

Perfusion technique. After an overnight fast, each rat was placed in a restraining cage and the jejunal segment was constantly perfused via the proximal catheter, using a syringe pump (Harvard Apparatus Inc., Millis, MA). The perfusion rate was $0.4 \mathrm{ml} / \mathrm{min}$ and the basic perfusion solution consisted of a $0.06 \mathrm{M}$ phosphate buffer $(\mathrm{pH}$ 6.6, the optimum $\mathrm{pH}$ of maltase, see references 15,16$)$. The solution also contained $\left[{ }^{3} \mathrm{H}\right]$ polyethylene glycol (PEG, 400,000 dpm/ml), $100 \mathrm{mg} / \mathrm{dl}$ PEG, and maltose at concentrations of 2.9, 14.6, 36.5, 73.1, 146, or 292 $\mathrm{mM}$ plus $\left[{ }^{14} \mathrm{C}\right]$ maltose $(40,000 \mathrm{dpm} / \mathrm{ml})$. Sodium chloride was added to adjust the osmolity of maltose plus $\mathrm{NaCl}$ to $300 \mathrm{mosmol} / \mathrm{kg}$.

The infusate solution was maintained at $37^{\circ} \mathrm{C}$ during the infusion, and at least four times the segment's volume was infused before the collection of samples via gravity drainage. Perfusate samples were boiled for 2 min immediately after collection to prevent the possibility of further hydrolysis in the collected samples. The hydrolysis rate of each maltose concentration was determined in 5-8 rats.

To determine the volume of the perfused segment, at the end of nine perfusion studies, a $0.1-\mathrm{ml}$ bolus of phenolsulfonphthalein was instilled into the infusion catheter and a series of 10 -s collections of perfusate were analyzed for phenolsulfonphthalein concentration by spectrophotometry (DU-70 spectrophotometer; Beckman Instruments, 
Inc., Fullerton, CA). The volume of luminal contents was determined via analysis of the outflow concentration profile of phenolsulfonphthalein using the Stewart-Hamilton formula (7). Eight of the nine studies yielded interpretable outflow curves, and the volume of luminal fluid in these studies averaged $1.5 \mathrm{ml} \pm 0.4 \mathrm{ml}$ for the 15 -cm segment.

Quantitation of villous architecture. Because villous architecture varies with the degree of distension of the intestine (8), it was necessary to examine the intestine at roughly the same degree of distension as was present in the perfused intestine. In three rats, $15-\mathrm{cm}$ segments of jejunum were isolated between ligatures and isotonic saline was perfused through the segment to remove debris. The segment was then stripped free of fluid and filled with $1.5 \mathrm{ml}$ of saline (the volume determined to be present in the perfused segments, as described above), both ends of the segment were occluded and the distended segment was placed in $10 \%$ formalin fixative. Segments of jejunum were sectioned longitudinally or perpendicular to the long axis of the villi in $7-\mu \mathrm{m}$-thick sections. The dimensions of the villi and the intervillous space were then determined via microscopical examination using a calibrated eyepiece. Approximately 20 villi were examined per section.

In vitro studies. After completion of the series of in vivo measurements of maltose hydrolysis, five animals were anesthetized with pentobarbital, the abdomen was opened, and the entire $15-\mathrm{cm}$ segment of perfused jejunum was removed. All mucosa of the segment was stripped off with a scalpel blade, diluted in a cold 0.06-M phosphate buffer at $\mathrm{pH} 6.6$, and homogenized using a tissue grinder. The maltase activity of an aliquot of the homogenate was determined in a vigorously stirred solution containing $146 \mathrm{mM}$ maltose in a $0.06-\mathrm{M}$ phosphate buffer $\left(\mathrm{pH} \mathrm{6.6)}\right.$ ) at $37^{\circ} \mathrm{C}$. The in vitro $\mathrm{V}_{\max }$ of the entire segment was then calculated from the glucose released during a $10 \mathrm{~min}$ incubation. In additon, to determine the $K_{\mathrm{m}}$ of the enzyme, aliquots of the homogenized mucosa were incubated with vigorously stirred solutions containing $2.5,5,10,20$, and $50 \mathrm{mM}$ maltose at $\mathrm{pH} 6.6$ and $37^{\circ} \mathrm{C}$. Sufficient $\mathrm{NaCl}$ was added to the buffers such that the sum of the osmolality of maltose plus $\mathrm{NaCl}$ equaled $300 \mathrm{mosmol} / \mathrm{kg}$. The $K_{\mathrm{m}}$ was then determined from a double-reciprocal plot of hydrolysis rate versus maltose concentration. No statistically significant difference in maltase activity was observed when activity was determined over a $\mathrm{pH}$ range of 6.6-7.2.

Analyses. Glucose was measured via a glucose oxidase technique and radioactive compounds were measured via scintillation counting with appropriate correction for crossover of the isotopes.

Calculations. The rate of maltose hydrolysis by the $15-\mathrm{cm}$ jejunal segment was determined from the sum of the ${ }^{14} \mathrm{C}$ disappearing from the segment and the glucose present in the perfusate:

Rate of maltose hydrolysis $=$

$$
\begin{aligned}
& \frac{(\text { Inf rate })\left(\left[{ }^{14} \mathrm{C}\right] \mathrm{Inf}\right)}{\text { ( s.a. maltose })}-\frac{\left[{ }^{14} \mathrm{C}\right] \text { perf }}{(\text { s.a. maltose })}- \\
& 0.5 \text { glucoseperf Inf rate } \frac{[\text { PEG }] \text { Inf }}{[\text { PEG] perg }}
\end{aligned}
$$

where [ ] inf and [ ]perf refer to concentrations of compounds in fluid entering and leaving the segment, respectively, and s.a. maltose refers to specific radioactivity of maltose $(\mathrm{cpm} / \mathrm{mmol})$. The logarithmic mean luminal concentration of maltose was calculated from:

$\log$ mean concentration $=\frac{([\text { malt }] \text { perf }-[\text { malt }] \text { inf })}{\text { In }([\text { malt }] \text { perf } /[\text { malt }] \text { inf })}$

Maltose hydrolysis rates for maltose concentrations of $>146 \mathrm{mM}$ were predicted, assuming Michaelis-Menten kinetics, from the observed hydrolysis rates for infusate maltose concentrations up to 146 $\mathrm{mM}$ from a plot using the Eadie-Hofstee plot:

Hydrolysis rate vs hydrolysis rate/log mean [maltose]
The predicted maltose hydrolysis rate for various maltose concentrations, assuming no unstirred layer, was predicted from the $\mathrm{V}_{\max }$ and $K_{\mathrm{m}}$ using the standard formula:

Hydrolysis rate $=\frac{\left.\mathrm{V}_{\max } \text { [maltose }\right]}{[\text { maltose }]+\mathrm{K}_{\mathrm{m}}}$

Model of villous architecture and calculations employed to predict maltose hydrolysis. The villi were approximated by rectangular prisms with cross sectional dimensions of $(u) \times(v)$ and a length $(d)$ from the villous crypt to the tip. The width $(w)$ of the intervillous space was set at $15 \mu \mathrm{m}$ (as determined from analysis of histological sections). It was assumed that there is an unstirred layer of thickness $L$ over the villous tips, and this unstirred fluid extends throughout the intervillous space. Maltase is located on the villous tips, and, in decreasing concentration, along the sides of the villus. It is assumed that the rate of maltose hydrolysis per unit villous surface area $(q)$ can be described by Michaelis-Menten kinetics:

$q=\mathrm{V}(X) \frac{C}{\mathrm{~K}_{\mathrm{m}}+C}$

where $V(X)$ is the $\mathrm{V}_{\max }$ per unit area of villous surface and is a function of $X$, the distance from the villous tip ( $X=0$ is at the tip and $X=d$ at the base of the villus), and $C$ is the maltose concentration at the brush border hydrolytic site. From the measurements of Nordstrom, et al. (9), $V(X)$ (i.e., maltase activity) decreases roughly linearly from the tips to the base, and it will be assumed that this activity can be described by:

$V(X)=V_{0}[1-(1-g) X / d]$

where $V_{0}$ is the value of $\mathrm{V}_{\max }$ at the villous tip, and $g$ is the ratio of $\mathrm{V}_{\max }$ at the base to that at the tip $(g \approx 0.4)$. The $K_{\mathrm{m}}$ is known from in vitro measurements of maltose hydrolysis and should be the same at all positions along the villus. Since the intervillous width $(w)$ is about $15 \mu \mathrm{m}$, the maximal distance that separates solute and epithelium in the intervillous space is $7.5 \mu \mathrm{m}$. If the intervillous space is considered to be a square column with cross sectional dimensions of $15 \times 15 \mathrm{~cm}$, $50 \%$ of solute molecules will be $<2.5 \mu \mathrm{m}$ from epithelium. Thus, it can be assumed that diffusion is sufficiently fast to make the concentration uniform in the direction parallel to the serosal surface, and that concentration varies only in the perpendicular direction. In addition, the analysis includes the possibility of a fluid secretion, either from the villous crypts or from just the villous tips.

With these assumptions, the following mathematical description of the maltose hydrolysis can be derived: define $C_{\mathrm{L}}$ as the mean concentration in the well-stirred luminal region, and $C_{\mathrm{t}}$ as the concentration at the tips of the villus (after diffusion through the unstirred layer of thickness $L$ ). The concentration in the intervillous space at the level of the tips $(X=0)$ will also be $C_{\mathrm{t}}$. There will be a steady state concentration gradient $[\mathrm{C}(\mathrm{X})]$ in the intervillous space, established by the diffusion, convection, and hydrolysis of maltose. This intervillous concentration is then described by the nonlinear differential equation:

$D A_{\mathrm{s}} \frac{\mathrm{d}^{2} C}{\mathrm{~d} X^{2}}-A_{\mathrm{s}} f \frac{\mathrm{d} C}{\mathrm{~d} X}=S V(X) \frac{C}{C+K_{\mathrm{m}}}$

where $D$ is the maltose diffusion coefficient $\left(0.67 \times 10^{-5} \mathrm{~cm}^{2} / \mathrm{sec}\right), A_{\mathrm{s}}$ is the fractional cross sectional area of the intervillous space (area/ $\mathrm{cm}^{2}$ of serosal area) and $S$ is the lateral villous surface area/villus length per $\mathrm{cm}^{2}$ serosal area. The constant $f$ is the fluid flow velocity produced by secretion from the crypts at the base of the intervillous space. In the intervillous space, $f=J_{\mathrm{v}} / A_{\mathrm{s}}$ where $J_{v}$ is the rate of fluid secretion in units of milliliters/second per $\mathrm{cm}^{2}$ of serosal area. In the unstirred layer above the tips, $f=J_{v}$. 

tion:

Eq. 7 is solved subject to the following two-point boundary condi-

At $X=0: \quad C=C_{\mathrm{t}}$

At $X=d: D \frac{\mathrm{d} C}{\mathrm{~d} X}-f C=0$

The rate of hydrolysis (per $\mathrm{cm}^{2}$ serosal area) by the villous tips $\left(Q_{\mathrm{t}}\right)$ is equal to:

$Q_{\mathrm{t}}=A_{\mathrm{t}} \mathrm{V}_{0} \frac{C_{\mathrm{t}}}{K_{\mathrm{m}}+C_{\mathrm{t}}}$

where $A_{\mathrm{t}}$ is the fractional cross sectional area of the villous tips. In the steady state, the rate of maltose hydrolysis by the sides of the villi $\left(Q_{\mathrm{s}}\right)$ is equal to the net rate that maltose enters the intervillous space, which is equal to the diffusive and convective terms at the villous tips:

$Q_{\mathrm{s}}=-A_{\mathrm{s}} D\left(\frac{\mathrm{d} C}{\mathrm{~d} x}\right)_{x=0}+A_{\mathrm{s}} \mathrm{f} C_{\mathrm{t}}$

If the convective flow in the intervillous space (i.e., f) is negligible, the convective term, $A_{\mathrm{s}} \mathrm{f} C_{\mathrm{t}}$, becomes zero and maltose entry into the intervillous is determined solely by the diffusive component.

The total rate of maltose hydrolysis $(Q)$ is equal to:

$\mathrm{Q}=\mathrm{Q}_{\mathrm{t}}+\mathrm{Q}_{\mathrm{s}}$.

The variables $Q, Q_{\mathrm{t}}$, and $Q_{\mathrm{s}}$ are all per $\mathrm{cm}^{2}$ of serosal surface. At very high concentrations, when all the maltase is saturated, the maximum rate of maltase hydrolysis $\left(Q_{\max }\right)$ is equal to the hydrolysis at the villous tips plus the integral of the maximum rate on the sides of the villus:

$Q_{\max }=V_{0}\left[A_{\mathrm{t}}+S d(1+f) / 2\right]$

The above derivation has been based on the assumption that the maltose concentration at the villous tips $\left(C_{\mathrm{t}}\right)$ is known (see Eq. 8), when, in reality, only the luminal concentration $C_{\mathrm{L}}$ is known. The concentration $(C)$ in the unstirred layer between the tips and well-mixed luminal region is described by the differential equation:

$D \frac{d C}{d X}-f C=Q$

where $Q$ is the rate of metabolism (Eq. 11). Solving Eq. 13 (using the boundary condition that $C(0)=C_{\mathrm{L}}$ ) for the concentration at the villous tips:

$C_{t}=\left(C_{L}-\frac{Q}{f}\right) e^{\frac{f L}{D}}+\frac{Q}{f}$

This equation can then be solved for $Q$ as a function of $C_{\mathrm{t}}$ and $C_{\mathrm{L}}$ :

$Q=f\left(C_{t}-C_{L} e^{\frac{f L}{D}}\right) /\left(1-e^{\frac{f L}{D}}\right)$

The solution to the set of Eqs. 7-15 is obtained as follows. First, an initial value of $C_{\mathrm{t}}$ is assumed and the two-point differential Eq. 7 is solved by a Runge-Kutta shooting method (10). This solution yields the value of $\mathrm{d} C / \mathrm{d} x$ at $X=0$ from which $Q$, defined by Eq. 11, can be determined and compared with the $Q$ defined by Eq. 15 . Then, by a trial and error (interpolation) procedure, $C_{\mathrm{t}}$ is adjusted (and the differential equation is solved) until the $Q$ from Eqs. 11 and 15 are equal (to within a fractional error of $10^{-4}$ ), yielding the final solution for $Q$. These equations are for the case where the secretion is from the villous crypts. If the secretion is from just the tips, then $f=0$ in Eqs. 7 and 8 and $f$ is unchanged in Eq. 15.

The parameters $A_{\mathrm{t}}, A_{\mathrm{s}}$ and $S$ can be related to the values of $u, v$, and $w$ defined above. Define $N$ as the number of villi per $\mathrm{cm}^{2}$ serosal area. Then $N=1 /[(u+w)(v+w)], A_{\mathrm{t}}=N u v, A_{\mathrm{s}}=1-A_{\mathrm{t}}$ and $S=$ $2 N(u+v)$. All measurements of maltose hydrolysis were made using a $15-\mathrm{cm}$ length of intestine. These measurements are converted into values that are normalized to $1 \mathrm{~cm}^{2}$ of serosal area (the units of the above theoretical expressions) by multiplying by the experimental value of the area per unit length, which was determined from the volume $(1.5 \mathrm{ml})$ of the $15-\mathrm{cm}$ length of intestine. The value of the parameter $V_{0}$ was determined from the in vitro measurements of the maltose hydrolysis by the serosal surface. All the maltase in a $15-\mathrm{cm}$ loop was extracted and the true value of $\mathrm{V}_{\max }$ (units: milligrams/second per $\mathrm{cm}^{2}$ serosal surface) was determined. This value of $\mathrm{V}_{\max }$ should be equal to the $Q_{\max }$ (Eq. 12), and Eq. 12 can be solved for $V_{0}$. The other experimental measurement that is need is $C_{\mathrm{L}}$, the mean concentration in the well-stirred lumen. This concentration drops as the infusate traverses the segment, and the value employed in the theoretical equations was the logarithmic mean concentration determined from Eq 2.

The predicted hydrolysis rate for the special case of a uniform unstirred layer of thickness $L$ over the tips and sides of the villi was determined as follows:

For the villous tips, the rate of diffusion through the unstirred layer can be set equal to the rate of hydrolysis by the maltase on the villous tips:

$Q_{t}=V_{o} C_{t} /\left(K_{m}+C_{t}\right)=D A_{t}\left(C_{o}-C_{t}\right) / L$

This can be written in the form of a quadratic equation for $C_{\mathrm{t}}$ which can then be found analytically. Knowing $C_{t}$, the rate of hydrolysis can be obtained. A similar procedure can be used for the sides of the villi, except that the value of $\mathrm{V}_{\max }$ varies as a function of distance from the tip, and an integration over the surface is required.

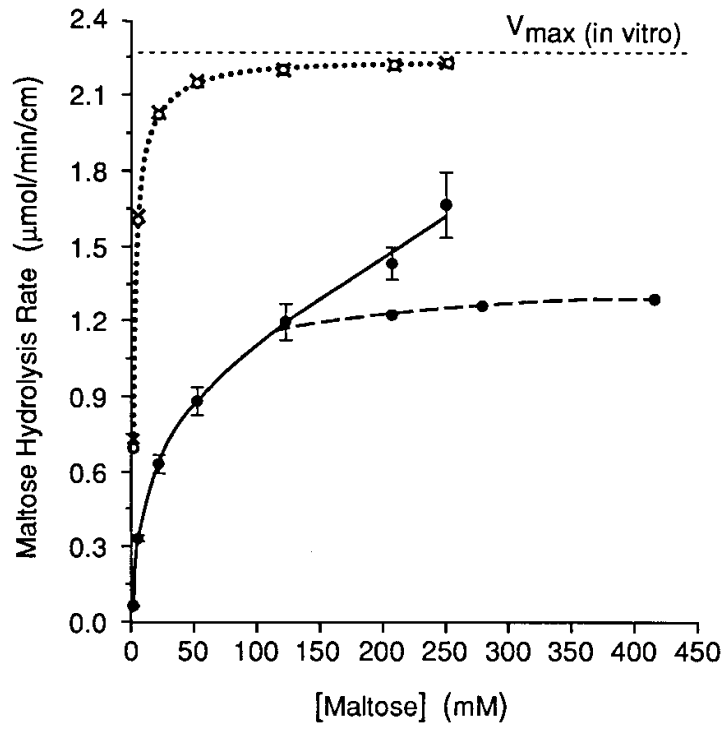

Figure 1. Observed (mean \pm SEM) and predicted maltose hydrolysis rates for varying logarithmic mean concentrations of maltose in the rat jejunum: hydrolysis rate observed in perfused jejunum (-); hydrolysis rate predicted for maltose concentrations $>146 \mathrm{mM}$ when Michaelis-Menten kinetics were employed with maltose concentrations $<146 \mathrm{mM}(\bullet---\bullet)$; hydrolysis rates predicted with $0(\mathrm{X} \cdots \mathrm{X})$ or $7 \mu \mathrm{m}(\bigcirc \cdots \bigcirc)$ unstirred layer covering entire epithelium (values virtually superimposable). The $\mathrm{V}_{\max }$ observed in vitro, $2.3 \mu \mathrm{mol} / \mathrm{min}$ per $\mathrm{cm}$ (dashed line) is about $80 \%$ higher than the $\mathrm{V}_{\max }$ predicted from Michaelis-Menten kinetics $(1.3 \mu \mathrm{mol} / \mathrm{min}$ per $\mathrm{cm})$. 


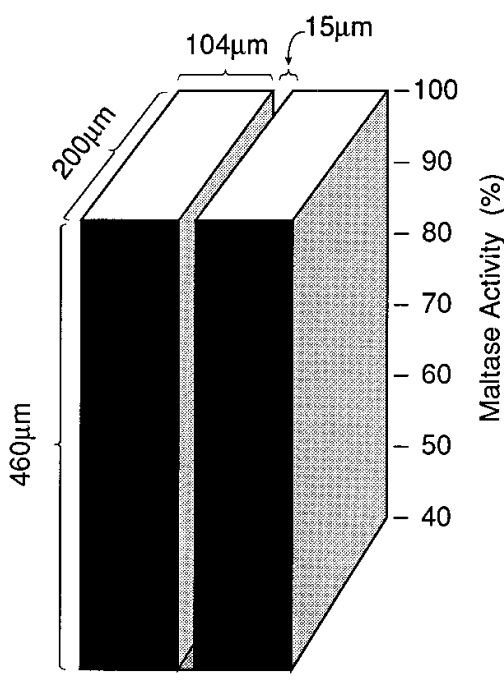

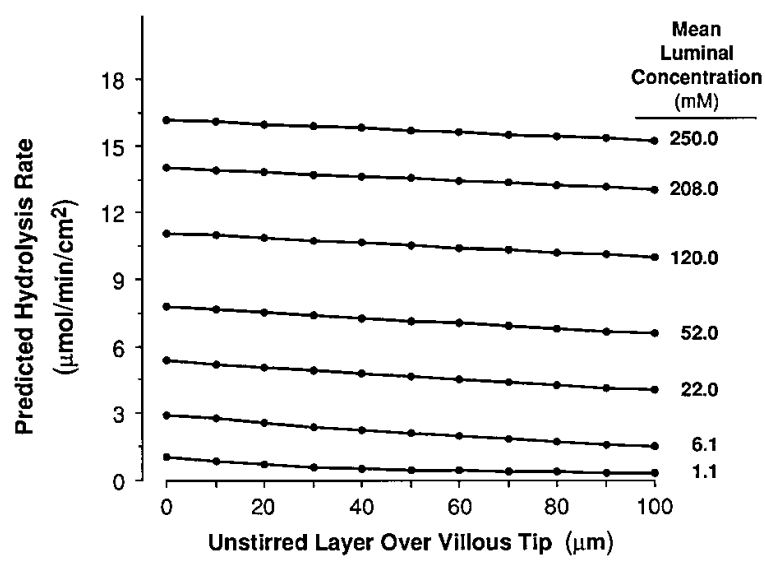

Figure 2. Parameters of the villous model used to predict maltose hydrolysis.

\section{Results}

Fig. 1 shows the observed in vivo maltose hydrolysis rates (mean \pm SEM) for each logarithmic mean luminal maltose concentration. The in vitro studies yielded values for the $K_{\mathrm{m}}$ and $\mathrm{V}_{\max }$ of maltase activity of $2.4 \pm 0.061 \mathrm{mM}$ and $34 \mu \mathrm{mol} / \mathrm{min}$ for the entire $15-\mathrm{cm}$ jejunal segment.

The dimensions of the jejunal villi (mean \pm 1 SD) averaged $104 \pm 11 \mu \mathrm{m}$ by $199 \pm 14 \mu \mathrm{m}$ by $460 \pm 46 \mu \mathrm{m}$ in length. The intervillous space averaged $15 \pm 4 \mu \mathrm{m}$ in width.

The villous model was then solved for the following "standard" set of parameters, determined from experimental measurements and literature values: the villous cross section was $u=104$ by $v=200$ microns, the villous length was $d=460 \mathrm{mi}-$ crons, and the intervillous width was $w=15$ microns (see Fig. 2 ). In this model, the villous tips represent $57 \%$ of the cross sectional area of the mucosal surface and $7.88 \%$ of the total villous surface. A $K_{\mathrm{m}}$ of $2.4 \mathrm{mM}^{\mathrm{w}}$ as obtained from the in vitro measurements. The ratio of $\mathrm{V}_{\max }$ at the base to the tip of the villus was chosen as $g=0.4$ on the basis of Nordstrom et al. (9). The in vitro $V_{\max }$ per $15 \mathrm{~cm}$ of intestine was $35 \mu \mathrm{mol} / \mathrm{min}$. For this standard model, it was assumed that there was no secretion $\left(J_{\mathrm{v}}=0\right)$. Thus, all the model parameters can be determined from direct experimental measurements with the exception of $L$ (unstirred layer thickness) which is treated as an adjustable parameter. Fig. 3 shows that the theoretically predicted rate of hydrolysis for this standard parameter, set as $L$, was varied from 0 to $100 \mu \mathrm{m}$. Increasing the unstirred layer over the tips resulted in an appreciable percent reduction in hydrolysis rate only at the lowest maltose concentrations. Thus, the hydrolysis rates observed at the lowest concentrations were the primary determinants of the unstirred layer over tips that provided the best fit of the model to the observed data. For example, the predicted hydrolysis rates at the lowest mean luminal maltose concentration $(1.1 \mathrm{mM})$ was 0.11 $\mu \mathrm{mol} / \mathrm{min}$ per $\mathrm{cm}^{2}$ for zero unstirred layer and $0.027 \mu \mathrm{mol} / \mathrm{min}$ per $\mathrm{cm}^{2}$ for a $100-\mu \mathrm{m}$ unstirred layer, a nearly fourfold difference. In contrast, at the highest mean luminal concentration (281 $\mathrm{mM}$ ), zero and $100-\mu \mathrm{m}$ unstirred layers over the tips yielded predicted hydrolysis rates of about $1.6 \mu \mathrm{mol} / \mathrm{min}$ per $\mathrm{cm}^{2}$ and $1.5 \mu \mathrm{mol} / \mathrm{min}$ per $\mathrm{cm}^{2}$, a difference of less than $10 \%$. The best fit of the predicted to the observed hydrolysis data

Figure 3. Maltose hydrolysis rates predicted for model for varying logarithmic mean concentrations of maltose using the standard set of parameters (see text) and an unstirred layer over the villous tips varying in thickness from 0 to $100 \mu \mathrm{m}$.

was provided by an unstirred layer of about $20-\mu \mathrm{m}$ thickness over the villous tips. As shown in Fig. 4, with this layer, hydrolysis rates predicted by the model very closely approximated the observed rates over the entire 100 -fold range of infusate maltose concentrations.

Fig. 5 shows, for each logarithmic mean luminal maltose concentration, the predicted distance that maltose would have to diffuse to achieve $50 \%$ and $90 \%$ of the observed hydrolysis rates. These distances increased as the luminal concentration of maltose increased.

Fig. 6 shows the predicted hydrolysis rates for the model when one of the parameters (villus length, width of intervillous space, maltase concentration gradient on the villus, or crypt secretion) in the standard set was varied, keeping all other values constant.

In Fig. 1, the observed hydrolysis rates can be compared with those predicted if there were no unstirred layer or a $7-\mu \mathrm{m}$ unstirred layer covering the entire mucosa. Nearly identical hydrolysis rates were observed for a 0 - and a $7-\mu \mathrm{m}$ unstirred layer, and in both these situations, saturation of maltase hydrolysis occurred at much lower concentrations than was observed.

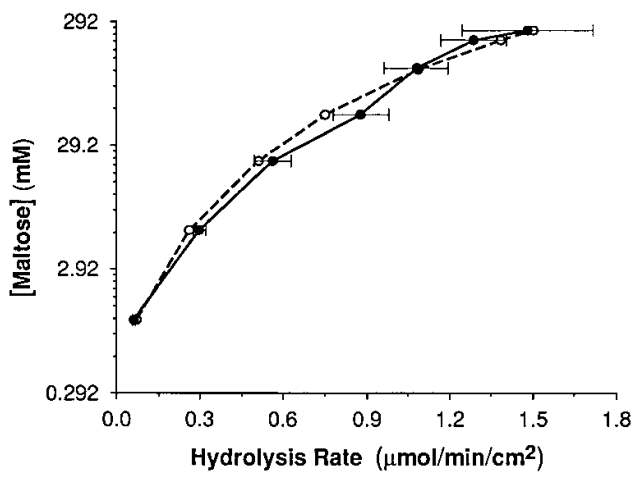

Figure 4. Comparison of the mean \pm SEM of observed hydrolysis rates $(--)$ and values predicted from the model $\left(\mathrm{O}_{-} \mathrm{O}\right)$ for varying logarithmic mean concentrations of maltose using a $20-\mu \mathrm{m}$ thick unstirred layer over the villous tips. 


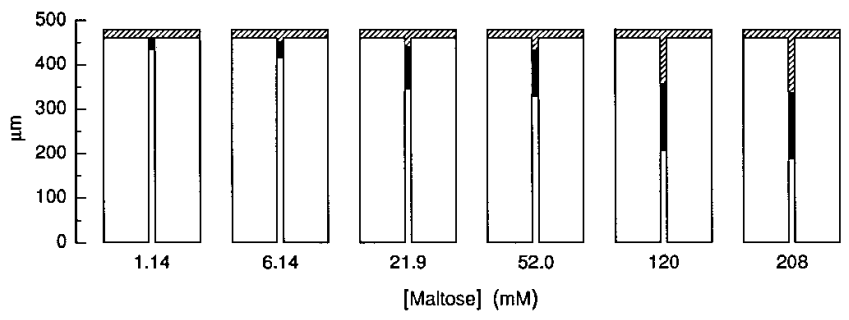

Figure 5. Predicted diffusion distance to achieve $50 \%$ of total hydrolysis (diagonal stripes) and additonal diffusion distance to achieve $90 \%$ of total hydrolysis (solid bar) for varying logarithmic mean luminal maltose concentrations.

The hydrolysis rates for infusate concentrations of $146 \mathrm{mM}$ or less were used to predict the hydrolysis rates for maltose concentrations greater than $146 \mathrm{mM}$ based on the assumption of Michaelis-Menten kinetics. As shown in Fig. 1, Michaelis kinetics predicted that hydrolysis should be nearly saturated at a luminal maltose concentration of $146 \mathrm{mM}$, whereas, the observed hydrolysis rates continued to rise as the infusate maltose concentration was increased to $234 \mathrm{mM}$ and $292 \mathrm{mM}$. The $\mathrm{V}_{\text {max }}$ measured in vitro $(2.3 \mu \mathrm{mol} / \mathrm{min}$ per $\mathrm{cm})$ was about $80 \%$ higher than the $\mathrm{V}_{\max }$ estimated from Michaelis-Menten kinetics $(1.3 \mu \mathrm{mol} / \mathrm{min}$ per $\mathrm{cm})$.

\section{Discussion}

The rate of jejunal transport or surface hydrolysis of a luminal solute is a function of the kinetics of the absorptive or hydrolytic process and the concentration of solute at the brush border. In the early 1970s, Dietchsy et al. (1) drew attention to the existence of unstirred luminal fluid adjacent to the mucosa that creates a diffusion barrier to more centrally located solute. As a result, solute concentration at the brush border may be only a small fraction of that in bulk luminal contents.

Most previous studies of the influence of the aqueous barrier on absorption have used a model in which a flat, fixed surface of functional epithelium is separated from solute in bulk luminal contents by an unstirred aqueous layer of uniform thickness (2-6). Use of this model has led to multiple attempts to elucidate a single thickness of unstirred fluid (the "unstirred layer") through which all solutes must diffuse to reach the brush border.

The goal of the present study was to use maltose hydrolysis measurements to elucidate the true in vivo interaction that exists between the unstirred layer and the villous epithelium of the rat jejunum. The methodology employed used the concept that the rate of absorption or surface hydrolysis of luminal solutes is a function of the kinetics of the absorptive or hydrolytic process and the concentration of solute at the brush border. Knowledge of the kinetics of the process makes it possible to model the appropriate aqueous diffusion barrier, (i.e., the barrier that would yield the brush-border concentrations that would make possible the observed absorption or hydrolysis rates). The accuracy of the technique requires that the kinetics, determined in vitro, where efficient mixing eliminates the unstirred layer effect, accurately reflect the in vivo situation. For this reason, we elected to employ the hydrolysis rate of maltose, as opposed to the absorption rate of a solute such as glucose, for these studies. The in vivo kinetics of this enzyme can be accurately and reproducibly determined by in vitro studies. In contrast, solute transport is a complex process, and kinetic data obtained in vitro tends to be highly variable and may not reflect the in vivo transport kinetics of the epithelial cells.

Modeling the interaction of the unstirred layer and the epithelial maltase first required a physical representation of these two entities. The dimensions of the villi and the intervillous space were determined from histological sections of intestinal

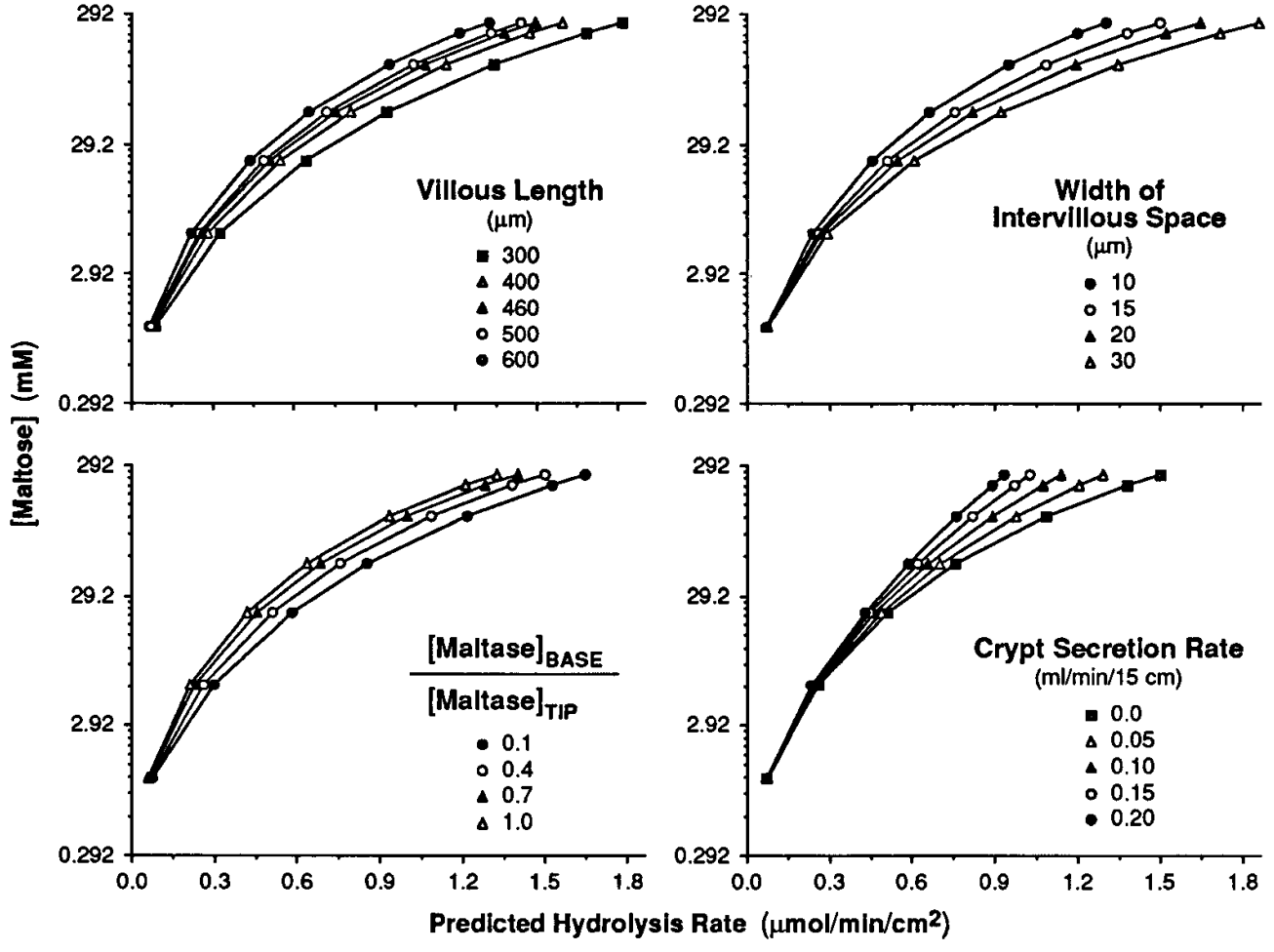

Figure 6. Influence of alteration of one of the parameters of the model (with the remainder of the parameters held constant) on predicted hydrolysis rates of maltose for varying logarithmic mean concentrations of maltose. 
segments distended to the same degree as was present in perfused intestine $(1.5 \mathrm{ml} / 15 \mathrm{~cm})$. As previously described, the villi were found to be leaf shaped rather than cylindrical (8), with dimensions of about $460 \mu \mathrm{m}$ in length by $200 \mu \mathrm{m}$ by 105 $\mu \mathrm{m}$. The intervillous space averaged about $15 \mu \mathrm{m}$ in width (see Fig. 2). These values are roughly comparable to those reported in the more elegant study of rat ileal mucosa by Harris et al. (8), although the jejunal villi were somewhat longer than reported for ileal villi. Maltase activity, determined from in vitro measurements of the $\mathrm{V}_{\max }$ and $K_{\mathrm{m}}$, was distributed over the villi of the model such that the activity declined from $100 \%$ at the villous tips to $40 \%$ at the base as reported by Nordstorm et al. (9). The potential aqueous diffusion barrier was modeled as totally unstirred water that extended from a variable distance over the villous tips throughout the extent of the intervillous space.

The goal was to determine what interaction of unstirred luminal fluid and maltase activity in the above model best explained the in vivo hydrolysis rates observed over a broad range of maltose concentrations. These hydrolysis measurements were obtained by constantly perfusing the jejunum of conscious animals via chronically implanted cannulas since previous studies have demonstrated that laparotomy and anesthesia markedly reduce luminal stirring in the rat (6).

Initially, we examined the possibility that the conventional model of the epithelium-unstirred layer relationship (i.e., a smooth layer of epithelium covered by a single thickness of unstirred fluid) was compatible with the observed data. There are two possible scenarios whereby this conventional model might reflect the situation in a villous epithelium: $(a)$ if solute absorption or hydrolysis occurred entirely at the villous tips after diffusing though a layer of unstirred fluid over the tips or $(b)$ if convection distributed solute throughout the intervillous space such that the solute was separated from the entire functional epithelial surface (tips and sides of villi) by the same thickness of unstirred fluid. The former possibility can be excluded since only $\sim 10 \%$ of the maltase activity of the segment was present on the villous tips, while $\sim 70 \%$ of the total maltase activity was required to account for the hydrolysis observed at high maltose concentrations. The second possibility also can be excluded. The intervillous space is only about $15-\mu \mathrm{m}$ wide; thus, convection into this space would virtually eliminate any unstirred layer separating cells along the sides of the villi from solute in this space. As shown in Fig. 1, maltase on a mucosa covered by unstirred layers of 0 or $7 \mu \mathrm{m}$ (the maximal possible distance separating solute and epithelium in the $15-\mu \mathrm{m}$-wide intervillous space) would be expected to saturate at much lower concentrations than was actually observed in the perfused jejunum.

It can be concluded from the above analysis that the epithelium lining the sides of the villi must play a major role in maltose hydrolysis and that maltose must reach this epithelium via diffusion down the longitudinal extent of the intervillous space rather than via convection. The simplest model of such a situation is a continuous unstirred water barrier beginning somewhere in the region of the villous tips and extending the length of the intervillous space. Using the parameters of our villous model, hydrolysis rates of maltose were predicted for such an unstirred water barrier, varying the thickness of fluid over the villous tips to determine the best fit to the observed data. As shown in Fig. 4, such an unstirred aqueous barrier beginning about $20 \mu \mathrm{m}$ above the villous tips yielded predicted maltose hydrolysis rates that rather accurately reflected ob- served hydrolysis rates over the entire 100 -fold range of infusate maltose concentrations. The need for a $20-\mu \mathrm{m}$ layer over the tips primarily was determined by the hydrolysis rates of the lowest concentrations of maltose; the predicted rates for high maltose concentrations were only minimally influenced by the thickness of the layer over the tips (see Fig. 3).

The good fit of the observed data to the model does not, of course, assure that all aspects of the model are correct. Other combinations of parameters might also have yielded data that fit the experimental findings. In addition, the parameters of our villous model represented average values for our rats, and some of these parameters were approximations. Variability in these parameters would be expected to exist at different intestinal sites in the same rat, between individual rats, and between species. Thus, while we believe that the model developed in this study provides qualitatively valid and useful insights into unstirred fluid-epithelial interactions, the quantitative predictions of the model are unlikely to have widespread applicability.

In distinct contrast to the conventional representation of functional epithelium as a fixed surface area covered by a fixed layer of unstirred fluid, in our villous model the functional epithelial area and the distance the solute must diffuse before hydrolysis are linked variables, both increasing as the efficiency of the absorptive or hydrolytic process decreases. At the lowest mean luminal maltose concentration $(1.1 \mathrm{mM}), 50 \%$ of the total hydrolysis occurs at the villous tip after diffusing $\sim 20 \mu \mathrm{m}$ through the unstirred layer that overlies the villous tips, and $90 \%$ hydrolysis occurs after diffusing another $20 \mu \mathrm{m}$ into the intervillous space (see Fig. 5). As the infusate concentration increases, maltase at the villous tips becomes saturated and the build up of maltose concentration at the tips drives diffusion deeper into the intervillous space. At a mean luminal maltose concentration of $208 \mathrm{mM}, 50 \%$ and $90 \%$ hydrolysis are achieved only after maltose diffuses $124 \mu \mathrm{m}$ and $270 \mu \mathrm{m}$ into the intervillous space (Fig. 5). Thus, efficiently absorbed or hydrolyzed solutes are largely handled at the villous tips after diffusing $\sim 20 \mu \mathrm{m}$. Very inefficiently handled solutes will diffuse throughout the extent of the intervillous space (about $460 \mu \mathrm{m}$ in the rat jejunum) exposing the entire villous surface to solute. The time-honored use of an unstirred "layer" to represent the aqueous diffusion barrier accurately portrays the true physiological situation only in the rare situation where the solute is very efficiently handled by the cells at the villous tips.

Previous attempts to measure the thickness of an unstirred layer in the human jejunum used several different techniques, and reported thicknesses have ranged from $600 \mu \mathrm{m}(11,12)$ to $40 \mu \mathrm{m}$ (13). As discussed above, the observed hydrolysis of low concentrations of maltose in the rat could not have occurred if the unstirred layer over the villous tips had been thicker than $\sim 20 \mu \mathrm{m}$. Analysis of the absorption of low glucose concentrations in man indicated that the observed absorption rates could not have occurred if the unstirred layer were greater than $40-\mu \mathrm{m}$ thick (13). In contrast, very slowly absorbed solutes would diffuse the entire length of the intervillous space, about $600 \mu \mathrm{m}$ in man. The maximal mean distance any solute might diffuse before transport would be one-half the length of the human intervillous space or about $300 \mu \mathrm{m}$.

The sensitivity of the model's predictions to potential alterations of one parameter (while holding the others constant) is shown in Fig. 6. The hydrolysis rate was relatively insensitive to physiological variations in the observed villous length. For 
example, increasing the villous length from the observed value of $460 \mu \mathrm{m}$ to $600 \mu \mathrm{m}$, or shortening it to $300 \mu \mathrm{m}$, decreased or increased hydrolysis rates by a maximum of about $15 \%$, respectively. Predicted hydrolysis rates were more sensitive to potential alterations in the diameter of the intervillous space, which averaged $15 \pm 4 \mu \mathrm{m}$. Decreasing the diameter to $10 \mu \mathrm{m}$ yielded maximal reductions of the predicted rate of hydrolysis of about $15 \%$, while increasing the diameter to $30 \mu \mathrm{m}$ yielded maximal increases of about $30 \%$. Since the primary effect of these parameter alterations was to alter the rate of delivery of maltose to enzyme on the sides of the villi, the effect was maximal at high luminal maltose concentrations. As shown in Fig. 6 , alterations of the assumed decline in maltase activity from tip $(100 \%)$ to base of the villus $(40 \%)$ had a minimal effect on the predicted rates of maltose hydrolysis.

Lastly, our model assumed negligible net fluid absorption or secretion in the intervillous space. Fluid secretion from the crypts would be expected to slow the rate of movement of solute down the intervillous space; whereas, net fluid absorption in the space would increase this rate of movement. As shown in Fig. 6, major reductions in hydrolysis rate would be expected to occur at high luminal maltose concentrations and relatively rapid secretion rates.

There are a number of physiological implications of the concept that hydrolysis (and presumably absorption) involves solute diffusion into the intervillous space. The conventional model of the epithelium and unstirred layer supposes a fixed number of active sites separated from bulk luminal solute by a fixed thickness of unstirred layer. As a result, this model saturates according to Michaelis-Menten kinetics. In contrast, the villous model is less readily saturated since more epithelial surface area is recruited as solute diffuses down the narrow corridor of the intervillous space. Thus, the $\mathrm{V}_{\max }$ predicted from Michaelis-Menten would be expected to underestimate the true $V_{\max }$ if our model of the unstirred water-epithelial interaction were correct. This was indeed the case in that the $\mathrm{V}_{\max }$ predicted from Michaelis-Menten kinetics for maltose concentrations of up to $146 \mathrm{mM}$ (the usual upper limit of concentration of maltose or glucose used for such predictions) was about $40 \%$ less than the true $\mathrm{V}_{\max }$ observed in vitro, and appreciably less than the rate actually observed at high concentrations of maltose, in vivo (see Fig. 1). It seems likely that previous estimates of $\mathrm{V}_{\max }$ of active transport of a variety of nutrients may have been similarly underestimated due to the inappropriate assumption that villous transport systems saturated according to Michaelis-Menten kinetics. For example, the failure of D-glucose transport to follow Michaelis-Menten kinetics has been attributed to passive transport of D-glucose. (3) This concept was supported by the finding of an appropriate rate of uptake of L-glucose, a presumed indicator of the paracellular transport of D-glucose. (3) However, a recent study suggested that L-glucose uptake was carrier mediated and not a reliable measure of paracellular transport of D-glucose (14). In addition, several recent reports have found minimal paracellular transport of hexoses $(14,15)$, suggesting that passive uptake explains the lack of saturation of uptake observed at high luminal D-glucose concentrations. It seems possible that this failure to saturate merely reflects the expected difficulty in saturation of a transport system located on the sides of the villi.

In 1977, Thomson and Dietschy (16) described an approach to estimating the intervillous unstirred layer effect in which they assumed that each cross sectional lateral surface of the villous was an equivalent flat surface with an effective unstirred layer that varied with the distance of that surface from the villous tip. This approach provides only an approximation of the true situation since, among other things, it does not account for the hydrolysis or absorption of solute during diffusion down the intervillous space. The author's calculations led them to conclude that Michaelis-Menten kinetics will not accurately predict active transport in a villous mucosa where effective surface area and unstirred layers are not constants.

The accurate prediction of absorption or hydrolysis rates is an important feature of a physiological model of the gut. Unfortunately, predictions of these rates from the villous model are far more complicated than is the case with the conventional assumption of Michaelis kinetics. Predictions using the villous model require knowledge of the kinetics along the entire extent of the villus, and these kinetics probably vary markedly from the bottom to the top of the villi.

Conventionally, functional absorptive surface area is considered to be a constant. Thus, transport rates can be influenced only via alteration of epithelial cell function or major increases of the unstirred layer over the villous tips. Neither of these mechanisms would be expected to be readily or rapidly adaptable to luminal conditions. In contrast, the present demonstration that hydrolysis (and presumably absorption) occurs along the sides of the villi suggests that functional surface area may be sensitively controlled via regulation of solute access to the intervillous space. Such regulation could be "passive" or "active." An example of passive regulation would be the widening of the intervillous space, with resultant increased access to the sides of the villi, that occurs with gut distention. In support of this concept is the demonstration that gut distention has been shown to increase the absorption rate of certain solutes (17). In contrast, minimal swelling of the villi would be expected to appreciably narrow the intervillous space and retard absorption. The gut is capable of copious secretion when exposed to a variety of stimuli, and this secretion is thought to originate in the crypts. Convection of fluid up the narrow confines of the intervillous space would far more efficiently limit solute access to mucosa than would a similar secretion originating from a flat mucosa of similar total surface area. On the other hand, the absorption of a solute such as glucose from the intervillous space would enhance water absorption from the space. The resultant convection of bulk luminal fluid into the space would increase the exposure of epithelium to luminal solutes, thus providing a mechanism for the well-known ability of glucose to enhance the absorption of a variety of other luminal solutes.

While rat villi are nonmotile, the villi of the dog, cat, and possibly man $(18,19)$ have been shown to have rhythmic contractile activity, and various luminal solutes have been shown to alter this contractility (20). It seems possible that villous contractions might serve to convect material into the intervillous space, thus providing a means by which the intestine could locally control its absorption rate.

Since absorptive surface area conventionally is considered to be constant, the influence of various manipulations on absorption rates usually have been attributed to alterations in the efficiency of transport by the individual epithelial cell. The present study suggests that changes in functional absorptive surface area due to varying access of solute to the intervillous space needs to be considered as a potential explanation for the influence of a variety of acute perturbations on absorption rate. 
Lastly, the combination of a thin unstirred layer over the villous tips and a thick unstirred barrier between the villi has implications for the intestinal handling of compounds that undergo surface hydrolysis such as maltose or limit dextrins. The thin layer over the tips permits the efficient hydrolysis and transport observed for low concentrations of these compounds. The requirement that high concentrations diffuse more deeply into the intervillous space before hydrolysis results in the release of most of the monomers at a site separated from bulk luminal contents by a relatively thick diffusion barrier. Thus, the monomers tend to be "trapped" in the intervillous space, rather than rapidly diffusing back into bulk luminal contents as would be the case with a thin unstirred layer. As a result, absorption and hydrolysis rates appear to be "linked" $(21,22)$ and large quantities of carbohydrate can be digested and absorbed with minimal increases in the osmolality and resultant volume of bulk luminal contents.

\section{Acknowledgments}

Supported in part by the Department of Veterans Affairs and the National Institutes of Diabetes and Digestive and Kidney Diseases grant RO1 DK 13309-25.

\section{References}

1. Dietschy, J.M., V.L. Sallee, and F.A. Wilson. 1971. Unstirred water layers and absorption across the intestinal mucosa. Gastroenterology. 61:932-934.

2. Levitt, M.D., A. Strocchi, and D.G. Levitt. 1992. Human jejunal unstirred layer: evidence for extremely efficient luminal stirring. Am. J. Physiol. 262 (Gastrointest. Liver Physiol.) 25:G593-G596.

3. Westergaard, H., K.H. Holtermuller, and J.M. Dietschy. 1986. Measurement of resistance of barriers to solute transport in vivo in rat jejunum. Am. J. Physiol. 250 (Gastrointest. Liver Physiol.) 13:G727-G735.

4. Winne, D. 1973. Unstirred layer, source of biased Michaelis constant in membrane transport. Biochim. Biophys. Acta. 298:27-31.

5. Smithson, K.W., D.B. Millar, L.R. Jacobs, and G.M. Gray. 1981. Intestinal diffusion barrier: unstirred water layer or membrane surface mucous coat?
Science (Wash. DC). 214:1241-1243.

6. Westergaard, H., and J.M. Dietschy. 1974. Delineation of the dimensions and permeability characteristics of the two major diffusion barriers to passive mucosal uptake in the rabbit intestine. J. Clin. Invest. 54:718-732.

7. Dow, P. 1956. Estimations of cardiac output and central blood volume by dye dilution. Am. J. Physiol. 36:77.

8. Harris, M.S., J.G. Kennedy, K.A. Siegesmund, and D.E. Yorde. 1988. Relationship between distention and absorption in rat intestine. Gastroenterology. 94:1164-1171.

9. Nordstrom, C., A. Dahlquist, and L. Josefsson. 1968. Quantitative determination of enzymes in different parts of the villi and crypts of rat small intestine, comparison of alkaline phosphatase, disaccharidases and dipeptidases. $J$. Histochem. Cytochem. 16:713-721.

10. Press, W.H., B.P. Flannery, S.A. Teukolsky, and W.T. Vetterling. 1986. Numerical Recipes. Cambridge University Press, New York. 550-559.

11. Frase, L.L., A.D. Strickland, G.W. Kachel, and G.J. Krejs. 1985. Enhanced glucose absorption in the jejunum of patients with cystic fibrosis. Gastroenterology. 88:478-484.

12. Read, N.W., D.C. Barber, R.J. Levin, and C.D. Holdsworth. 1977. Unstirred layer and kinetics of electrogenic glucose absorption in the human jejunum in situ. Gut. 18:865-876.

13. Levitt, M.D., J.K. Furne, A. Strocchi, B.W. Anderson, and D.G. Levitt 1990. Physiological measurements of luminal stirring in the dog and human small bowel. J. Clin. Invest. 86:1540-1547.

14. Schwartz, R.M., J.K. Furne, and M.D. Levitt. 1995. Paracellular intestinal transport of six-carbon sugars is negligible in the rat. Gastroenterology. 109: 1206-1213.

15. Fine, K.D., and J.L. Porter. 1993. Effect of D-glucose on intestinal permeability and its passive absorption in human small intestine in vivo. Gastroenterology. 105:1117-1125.

16. Thomson, A.D.R., and J.M. Dietschy. 1977. Derivation of the equations that describe the effects of unstirred water layers on the kinetic parameters of active transport processes in the intestine. J. Theor. Biol. 64:277-294.

17. Harris, M.S., and J.G. Kennedy. 1988. Relationship between distention and absorption in rat intestine. Gastroenterology. 94:1172-1179.

18. Kokas, E., and E.G. Ludany. 1934. Die hormonale regelung der darmzottenbewegung. II. Pfluegers Arch. Eur. J. Physiol. 234:182-186.

19. Kokas, E., and E.G. Ludany. 1933. Die hormonale regelung der darmzottenbewegung. I. Pfluegers Arch. Eur. J. Physiol. 232:293-298.

20. Womach, W.A., J.A. Barrowman, W.H. Graham, J.N. Benoit, P.R. Kvietys, and D.N. Granger. 1987. Quantitative assessment of villous motility. Am. J. Physiol. 252:G250-G256.

21. McMichael, H.G., J. Webb, and A.M. Dawson. 1967. The absorption of maltose and lactose in man. Clin. Sci. (Lond.). 33:135-145.

22. Gray, G.M., and F.J. Ingelfinger. 1966. Intestinal absorption of sucrose in man: interrelation of hydrolysis and monosaccharide production absorption. J. Clin. Invest. 45:388-398. 